Topic 4: Active Galactic Nuclei 


\title{
The remarkable AGN jets
}

\author{
Serguei Komissarov \\ School of Mathematics, University of Leeds, \\ Leeds LS29JT, United Kingdom \\ email: s.s.komissarov@leeds.ac.uk
}

\begin{abstract}
The jets from active galactic nuclei exhibit stability which seems to be far superior compared to that of terrestrial and laboratory jets. They manage to propagate over distances up to a billion of initial jet radii. Yet this may not be an indication of some exotic physics but mainly a reflection of the specific environment these jets propagate through. The key property of this environment is a rapid decline of density and pressure along the jet, which promotes its rapid expansion. Such an expansion can suppress global instabilities, which require communication across the jet, and hence ensure its survival over huge distances. At kpc scales, some AGN jets do show signs of strong instabilities and even turn into plumes. This could be a result of the flattening of the external pressure distribution in their host galaxies or inside the radio lobes. In this regard, we discuss the possible connection between the stability issue and the Fanaroff-Riley classification of extragalactic radio sources. The observations of AGN jets on sub-kpc scale do not seem to support their supposed lack of causal connectivity. When interpreted using simple kinematic models, they reveal a rather perplexing picture with more questions than answers on the jets dynamics.
\end{abstract}

Keywords. black hole physics, relativity, galaxies: jets, hydrodynamics, MHD, radio continuum: galaxies

\section{Introduction}

The terrestrial and laboratory gas and plasma jets are know to be susceptible to instabilities, which tend to destroy them over relatively short distances. This is particularly true for subsonic jets but also applies to supersonic jets. Although, supersonic jets are more resilient, the typical laboratory jets slow down and dissipate most of its kinetic energy over the distances which do not exceed a hundred initial jet radii. In contrast, the AGN jets appear to be much more robust. Indeed, the initial radius of an AGN jet is expected to be comparable to the gravitational radius of the central black hole, which is $r_{i} \sim 10^{14} \mathrm{~cm}$ for $10^{9} M_{\odot}$, and these jets can often be traced up to the distance of $100 \mathrm{kpc}$, which is about one billion of the initial jet radius. At first glance, this remarkable contrast between the AGN jets and their terrestrial counterparts suggests a dramatically different physics. After all, these jets are relativistic and produced by black holes. We cannot reproduce them in a physical laboratory. However, the results of numerous theoretical and computational studies suggest that this may not be the right conclusion.

The properties of relativistic fluid jets are found to be quite similar to those of nonrelativistic jets, when using proper parametrisation. For example, initially it was claimed that high-Lorentz-factor jets are less unstable. However, both the relativistic Mach number and the specific inertia (mass density in the lab frame) depend on the Lorentz factor (Konigl 1980, Komissarov \& Falle 1996) and hence the results of the instability studies are well in line with the known dependence of growth rates on the Mach number and density ratio for non-relativistic jets. Moreover, the jets from young stars, which are non-relativistic, show a similar ability to propagate over huge distances, not much different from AGN jets when measured in the units of initial jet radius. The magnetic field 
can suppress some non-magnetic instabilities but introduces new current-driven instabilities, which could be even more destructive given its predominantly toroidal (azimuthal) structure expected far away from the magnetic central engine.

The laboratory jets are approximately cylindrical (albeit with oscillations due to initial difference in pressure with the external gas) and most theoretical and numerical stability studies have been focused on cylindrical jets. This symmetry assumption allows to simplify the problem and helps to make it treatable by standard analytic techniques. The AGN jets are not cylindrical, with the half-opening angle in the region of few degrees. This may not seem a lot and at first suggests that the cylindrical approximation may be suitable. On the other hand, over the long length of the jets, this amounts to a huge increase in jet radius. The same applies to the stellar jets. Although the stability of expanding jets is not well studied, it has been argued that such an expansion has a stabilising effect (e.g. Moll et al. 2008).

\section{Causality and jet stability}

It helps to distinguish between local and global instabilities of jets. Global modes imply coherent motion on the scale of the jet radius. One typical example of such a global instability is the kink mode, where the whole jet moves sideways attaining a snake-like or a helical shape. Another is the pinch mode, where the jet radius varies independently of the azimuthal angle. Such instabilities are recognised as the most threatening to the jet survival, as they lead to strong interaction with the surrounding gas in the non-linear regime. Local instabilities are small scale motions. They may lead to local dissipation and mixing but do not endanger the jet integrity. Clearly, only the global instabilities are relevant to the issue of the astrophysical jets ability to propagate over very large distances.

The very nature of global instabilities implies a causal communication across the jet. Without such a communication it is impossible to support such highly-organised motions. In fluids, the communication speed is limited by the fundamental wave speeds, the sound speed in gas dynamics and the fast magnetosonic speed in MHD. For supersonic (super-fast-magnetosonic) flows, these waves cannot propagate upstream. In fact, they are confined to a downstream-pointing cone known as the Mach cone. A higher Mach number corresponds to a narrower Mach cone and hence a longer distance over which the jet becomes aware of an upstream perturbation. This explains why the growth rate of instabilities in cylindrical jets decreases with the Mach number. In expanding supersonic jets, the Mach number monotonically increases and hence the growth rate decreases along the jet. The instabilities become suppressed altogether when the typical opening angle of the Mach cone becomes smaller than that of the jet (This argument applies equally well to both relativistic and non-relativistic flows.).

Consider for example a freely-expanding non-relativistic unmagnetised supersonic jet. Such a jet is almost conical and propagates at constant speed. Its density $\rho \propto z^{-2}$, pressure $p \propto \rho^{\gamma}$, sound speed $a^{2} \propto p / \rho$ and the Mach angle $\theta_{m} \propto a$. From these we find $\theta_{m} \propto z^{1-\gamma}$ and hence the Mach angle is bound to eventually become less than the opening angle of the jet, leading to its global stability. Presumably, this simple argument was behind the assumption made in some early studies of kpc AGN jets that they emerge from AGN as cold conical flows (e.g. Falle 1991).

If a jet is not freely expanding but instead it is confined by an atmosphere with the power law pressure $p_{\text {ext }} \propto z^{-\kappa}$ then the ratio of the Mach angle and the jet opening angle $\theta_{m} / \theta_{j} \propto z^{(2-\kappa) / 2}$ (Porth \& Komissarov 2015). This indicates that $\kappa=2$ is a critical value, with jets becoming causally-disconnected for $\kappa>2$. Such causally-disconnected 

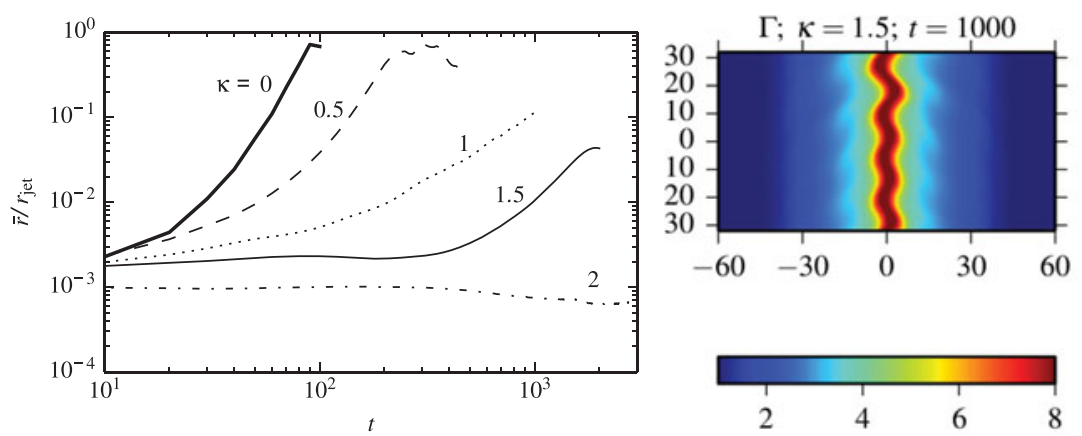

Figure 1. Left panel: Amplitude evolution of a kink-mode instability as observed in the simulations by Porth \& Komissarov (2015). The amplitude is defined as the sideways displacement of the jet barycentre measured in the units of the instantaneous jet radius. The time is measured in the units of $r_{0} / c$, where $r_{0}$ is the initial jet radius. Right panel: The unstable core of the jet in the atmosphere with $\kappa=3 / 2$. The image shows the Lorentz factor distribution.

jets are not necessarily freely expanding conical flows. Indeed, in a conical jet its pressure decreases as $p \propto z^{-2 \gamma}$, which is faster than that of the external gas if $\kappa<2 \gamma$. Hence for $2<\kappa<2 \gamma$ the jets must still be confined by the external pressure, which is now communicated to the jet interior by shock waves. However, these are high amplitude waves and not the small perturbations associated with instabilities, which suggests that for $\kappa>2$ jets are still globally stable. Relativistic and magnetic effects do not seem to alter this conclusion (Porth \& Komissarov 2015).

The central engines of AGN and stellar jets are dominated by compact objects, whose gravitational field has a strong impact on the distribution of surrounding gas. The overall effect is a rapid decrease of both its density and pressure away from the centre. For a polytropic atmosphere of central mass, one has $\kappa=\gamma /(\gamma-1)$, which is higher than 2 when $1<\gamma<2$. For a spherical adiabatic wind, $\kappa=2 \gamma$, which is also steeper than the critical one. A self-collimating magnetic wind can in principle deliver $\kappa<2$. For the Bondi accretion $\kappa=3 \gamma / 2$, which is still larger than 2 for $\gamma>4 / 3$. Thus, steep gradients of external pressure, bordering the critical value, are expected to be quite common close to the central engine. Unfortunately, we are still unable to measure the parameters of the external gas on the scales below $1 \mathrm{kpc}$. Instead, we have to rely mostly on indirect model-dependent estimates. Taken together, this circumstantial evidence suggests that $\kappa \simeq 2$ is indeed quite typical for the AGN environment (e.g. Phinney 1983, Begelman et al. 1984).

In order to study the effect of jet expansion on its stability, Porth \& Komissarov (2015) used periodic box simulations where the pressure of external gas was forced to decrease as a power function of time, similarly to what would have been seen in a frame moving radially at constant speed through a power-law atmosphere. The left panel of Fig. 1 shows the growth of a kink-mode perturbation in a moderately relativistic magnetised jet with a purely azimuthal magnetic field. One can see, that the growth rate decreases with $\kappa$ and the instability is totally suppressed for $\kappa=2$, in line with the expectations.

One characteristic property of magnetised jets is the development of a magneticallyconfined core which refuses to expand with the same rate as the rest of the jet and remains causally connected. Hence, this core can develop instabilities and gets destroyed even when the rest of the jet remains stable. In fact, this is an example of a local instability at work. The right panel of Fig. 1 shows how this instability develops in the simulations 
by Porth \& Komissarov (2015). Such local instabilities may power emission from jets without endangering their integrity.

\section{The Fanaroff-Riley division}

The issue of jet stability is often discussed in connection to the global division of extragalactic radio sources into two main classes according to the classification by Fanaroff \& Riley (1974). The main motivation behind this connection is based on the remarkable similarity in appearance between the kpc-scale jets (and tails) of some FR1-type sources (e.g. 3C31) and the unstable transonic jets produced in laboratory. These sources do not show any cocoons separating their jets from the external gas and in some cases this gas is observed in X-rays (e.g. Schreier et al. 1982 for M87). Furthermore, the theoretical model based on the idea of a turbulent flow confined by the external pressure and entraining significant amount of surrounding gas has successfully reproduced a number of the observational results (e.g. Bicknell 1984, Komissarov 1990, Laing \& Bridle 2002). Thus, we need to take seriously the possibility that some AGN jets become reconfined by the external gas pressure at the distances of $0.1 \div 1 \mathrm{kpc}$ from the central engine and develop instabilities. In fact, the optical observations of giant elliptical galaxies indicate that their stellar distribution flattens near the centre. The size of this central core depends on the galactic mass and is scattered around $1 \mathrm{kpc}$. At such distances, the gravitational potential of the central black hole is sub-dominant and the gas distribution is expected to reflect that of the stars. In fact, it is known that outside of the core the gas distribution does mimic the stellar one (Mathews \& Brighenti 2003). Hence, the X-ray gas of galactic coronas is likely to have a central core with flat pressure distribution which could be sufficiently high to reconfine the FR1-type jets.

The reconfinement process involves a strong shock, driven into the jet by the external pressure. It turns out (see Porth \& Komissarov 2015) that for a uniform external gas of pressure $p_{0}$ and an initially conical jet of kinetic power $L_{j}$, the reconfinement shock reaches the jet axis at the distance $z_{r, k p c} \approx 0.3\left(L_{j, 44} / p_{0,-9}\right)^{1 / 2}$, where the units are $1 \mathrm{kpc}$, $10^{44} \mathrm{erg} / \mathrm{s}$ and $10^{-9} \mathrm{dyn} / \mathrm{cm}^{2}$. Since $p_{0,-9} \approx 1$ is typical for the central parts of the Xray coronas of elliptical galaxies and for FR1 sources $L_{j, 44}<1$ (Cavagnolo et al. 2010), the reconfinement of FR1 jets inside the galactic cores seems very likely. On the other hand, the kinetic power of FR2 is significantly higher and the reconfinement process cannot be completed inside the cores. Outside, the pressure of the X-ray gas decreases rapidly again, $p \propto z^{-1.5 \pm 0.25}$ (Mathews \& Brighenti 2003). This slows down not only the reconfinement process but also the development of instabilities that follows. So it is reasonable to conclude that jets which manage to escape the core will remain as stable supersonic flows for much longer.

In fact, the observations show that FR1 and FR2 sources belong to different regions in the $P-L_{O}$ plane, where $P$ is the source radio power and $L_{O}$ is the optical luminosity of the host galaxy, with the dividing line $P^{F R} \propto L_{O}^{2}$ (Owen \& Ledlow 1994). Assuming that the critical jet power, $L_{j}^{F R}$, separating FR1 and FR2 sources is set by the condition $z_{r}=z_{c}$, where $z_{c}$ is the X-ray core radius, Porth \& Komissarov (2015) obtain $L_{j}^{F R} \propto p_{0} z_{c}^{2}$. Thus, the Owen-Ledlow result can be explained if the parameters of X-ray cores depend on the optical luminosity (mass) of the parent galaxy in an agreeable way. Unfortunately, the X-ray cores are rarely resolved. If, however, their radius scales in the same way as that of the stellar distribution then $z_{c} \propto L_{O}^{1.1}$ and if the gas temperature scales as the stellar velocity dispersion then $p_{0} \propto L_{O}^{-0.15}$ (Porth \& Komissarov 2015). This leads to $L_{j}^{F R} \propto L_{O}^{2.05}$, which is basically the same as observed, provided $P \propto L_{j}$. Overall, this is a very encouraging result. 
Contrary to being often described as the prototype FR1 source, 3C31 represents only a small sub-class of FR1 sources. Most of them have jets submerged into radio lobes, which makes them more like FR2 sources. The difference in that in FR2 sources the jets remain well collimated all the way and terminate in a very bright and compact hot spot near the outer edge of the radio lobe, whereas the jets of FR1 sources seem to "disappear" halfway through the lobe (de Ruiter et al. 1990). These observations show that the model of a naked jet has its limitations and a better model of FR-1 sources has to incorporate not only the conditions close to the AGN, where their jets are naked, but also the distant regions where these jets inflate radio-lobes.

The widely accepted current model of FR2 radio sources involves two jets surrounded by a light cocoon, which shields them from direct contact with the interstellar/intergalactic gas (Falle 1991, Komissarov \& Falle 1998, Kaiser \& Alexander 1997). This cocoon is filled with plasma which has been supplied by the jets during their previous activity and passed through the jet termination shocks, associated in the model with the leading hot spots of a FR2 source. This cocoon is over-pressured with respect to the external gas and drives a strong shock through it. The expansion of this cocoon is slow compared to its sound speed and hence its pressure distribution is more or less uniform.

Falle (1991) has shown that the cocoon pressure is always sufficient to reconfine the initially free conical AGN jet and turn it into a quasi-cylindrical flow whose length/radius ratio increases with time. Falle (1991) argued that eventually the cylindrical section becomes long enough for the jets to develop instabilities and become turbulent, now inside the cocoon. He proposed that this may result in the change in morphology from the FR2 to the FR1 type. However his 2D axisymmetric simulations could not confirm this prediction. The $2 \mathrm{D}$ simulations of jets with slab symmetry by Komissarov \& Falle (2003) did exhibit a kink-mode instability but the lobe structure still remained more or less unchanged. The expected transition has been achieved only in the very recent 3D hydrodynamic simulations by Massaglia et al. (2016) and RMHD simulations by Tchekhovskoy \& Bromberg (2016). It turns out that the 3D effects are critical in this problem. While an interesting and promising idea, it remains to be seen if it can explain the separation between FR classes in the Owen-Ledlow plane.

The analytical model of FR2 sources by Falle (1991) deals only with power-law density distributions of the external gas as this assumption leads to a self-similar solution. In the solution the whole jet is surrounded by a melon-shaped expanding cocoon. This naturally leads to a picture where the interstellar gas is evacuated from the parent galaxy. However, this is likely to be an over-simplification. From the theoretical viewpoint, an external gas distribution with flat central core introduces a characteristic length-scale to the problem and this is likely to alter the dynamics. Observationally, the two-lobed structure of FR-2 radio-sources, even at low radio frequency where the synchrotron losses are week (e.g. Kassim et al. 1996), suggests that the melon-shape of their cocoons is not generic. This conclusion is strengthened by the X-ray observations which show the presence of hot gas in the gap between the lobes of FR2 sources (Nulsen et al. 2015). Thus, it looks more likely that close to AGN the FR2 jets are "naked" and only further out they are cocooned.

\section{Complications}

The idea that the rapid expansion of AGN jets delays the onset of global instabilities until kpc scales offers a simple and attractive explanation to the most basic observed properties of extended extragalactic radio sources within the framework of well known physics. Yet we need to learn more about the properties of ISM and the dynamics of AGN 
jets on sub-kpc scales in order to see if these jets are indeed largely causally-disconnected on these scales.

Using the basic steady-state theoretical models, one may expect such flows to have a relatively simple structure. Not affected by global instabilities, they should be more or less straight. In the absence of strong internal dissipation of kinetic energy, their acceleration should be quite efficient and so we expect them to be highly supersonic. Even for the rather slow magnetic collimation-acceleration mechanism, the acceleration should be completed by the distances corresponding to the blazar zone, $z \lesssim 1$ pc (Komissarov et al. 2007). Their shape is not that well determined. Although, highly-magnetised causally-disconnected jets are almost conical (Komissarov et al. 2009), the AGN jet are not expected to remain highly-magnetised on the distances above one parsec. As to weakly-magnetised jets, we have already noted that they cannot be conical unless $\kappa>2 \gamma \dagger$. Assuming that the dissipation associated with the shocks which adjust the jet pressure to that of the external gas with $<2 \kappa<2 \gamma$ is weak and they can still be treated as adiabatic, we find that the jet radius $r_{j} \propto z^{\kappa / 2 \gamma}$. So the shape is still parabolic. Weak oscillations associated with stationary shocks may be superimposed on top of this parabolic shape throughout the jet length. We also expect to find $\theta_{j} / \theta_{m}>1$. For magetically-dominated flows $\theta_{m} \approx \sigma^{1 / 2} / \Gamma$, where $\sigma \gg 1$ is the relativistic magnetisation parameter, whereas for particle-dominated plasma $(\sigma \ll 1) \theta_{m} \approx \beta_{s} / \Gamma$, where $\beta_{s}=a / c$ is the sound speed. Hence the condition of causal disconnection reads as $\theta_{j} \Gamma>\sigma^{1 / 2}$ and $\theta_{j} \Gamma>\beta_{s}$ for these two regimes respectively.

The properties of AGN jets on sub-kpc can be studied using the VLBI method; normally down to about one parsec from the central engine. These observations allow to measure the jet apparent opening angle and the apparent velocity of individual components in the plane of the sky. To convert these into the actual values one has to know the angle between the jet and the line of sight. A number of more or less reasonable assumptions can be made to estimate the viewing angle. For individual sources they may lead to significant errors but for a large sample one expects to obtain statistically reliable results. Analysis of large samples has become the main approach of the current studies of VLBI-jets. Several groups studied the distributions of $\Gamma$ and $\theta_{j}$ using somewhat different models and techniques and they all agreed that $\theta_{j} \Gamma=0.1 \div 0.2$ (Jorstad et al. 2005, Pushkarev et al. 2009, Clausen-Brown et al. 2013). This means that the jets can be causally disconnected only if they are particle-dominated and cool, with the sound speed $\beta_{s} \leqslant 0.1$. This implies that no matter what is the main energy type of the jets near the central source by the distance of about one parsec it is converted into the kinetic energy of bulk motion. This is consistent with the conclusion reached by Komissarov et al. (2007) that the magnetic acceleration of AGN jets should be completed inside the blazar zone. The alternative mechanism of thermal acceleration is even faster, with $\Gamma \propto r_{j}$.

However, Lister et al. (2013) have found that in the MOJAVE sample the jet components identified at larger projected distance $z_{p}$ from the core tend to have higher apparent speed. Moreover, Homan et al.(2015) have shown that individual components move with predominantly positive acceleration at $z_{p}<4 \mathrm{pc}$ and predominantly negative accelerations at $z_{p}>30 \mathrm{pc}$. If these proper motions are indicative of the bulk flow velocity then in stark conflict with the current theoretical models the jet speed keeps increasing even on the scales well above $z=1 \mathrm{pc}$ and hence even at such large distances there is still plenty of free energy in the magnetic or thermal form to power this acceleration.

The M87 jets stands out from the rest by the fact that this galaxy is very close and the

$\dagger$ For very hot jets with $p \gg \rho c^{2}$ this condition reads $\kappa>4$ but jets which have reached their asymptotic speed cannot remain that hot. 
jet can be traced almost all the way from the event horizon to the kpc scales. According to the observations, there exist three distinct zones. In the near zone, which extends up to about $z=0.1 \mathrm{pc}\left(100 r_{s}\right)$, the jet shape is approximately parabolic with $r_{j} \propto z^{0.75}$ (Hada et al. 2013). Here the jet is still inside the AGN. The middle zone extends up $z=300$ pc. In this zone, the jet shape is a different parabola with $r_{j} \propto z^{0.56}$ (Hada et al. 2013). This shape change may indicate a change in the jet environment. Presumably, in the middle zone the jet is already surrounded by ISM of the parent galaxył. The far zone extends into kiloparsecs and the jet shape is almost conical there (e.g. Nakamura \&Asada 2014).

The apparent speed of individual features is seen to increase with distance in the middle zone from the Lorentz factor $\Gamma \approx 1$ at $z=50 \mathrm{pc}$ to $\Gamma \approx 6$ at $z=300 \mathrm{pc}$ (Asada et al. 2014). Kovalev et al.(2007) report even smaller speeds, below few percent of the speed of light, for $1 \mathrm{pc}<z<50 \mathrm{pc}$. Optical observations indicate that in the far zone the apparent speed of proper motion decreases with distance. However, the velocity pattern is extremely complicated, with very large local variations within individual knots (Meyer et al. 2013). If taken as measurements of the bulk speed of a quasi-steady jet, the results by Asada et al. (2014) and Kovalev et al.(2007) indicate that up to the distance $z \approx 50 \mathrm{pc}$ the jet is still subsonic! Indeed, in the magnetic model the fact that the jet eventually reaches $\Gamma \approx 6$ means that at the start of the acceleration zone the flow magnetisation parameter $\sigma>6$. This corresponds to the Alfvén speed $c_{a}>0.91 c$. In the thermal model, this means that at the start of the zone the jet plasma is ultra-relativistically hot with the sound speed $a \approx c / \sqrt{3}$. Both the numbers are high enough to imply a subsonic motion at $z<50 \mathrm{pc}$, which is also in stark conflict with our current understanding of AGN jets. The low speeds also imply the Doppler factor of order unity, which suggests that the M87 jet is intrinsically one-sided and hence rather exceptional. Interestingly and in conflict with the above results, Walker et al. (2008) report the apparent speed $\approx 2 c$ at $z \approx 1 \mathrm{pc}$. Perhaps the interpretation of VLBI observations is not that straightforward and the observed proper motions are poor indicators of the jet speed.

Idealised steady-state theoretical models of jets have a very simple structure, either entirely featureless or with few clearly identifiable reconfinement shocks. Even a quick glance at the high-resolution images of AGN jets reveal that their structure is much richer. Variable central engine, cloudy environment and instabilities are the most obvious factors which can be responsible for this. All these factors can strongly modify the jet dynamics and make the idealised models redundant. More adequate models can be based on advanced computer simulations which have to accommodate the 3D nature of AGN jets. In these models, the issue of non-thermal particle acceleration and emission has to be addressed with rigour for the sake of direct comparison with the observations. Although some may think that we have captured the essence of the AGN jets, observations keep asking us challenging questions about this remarkable astrophysical phenomenon and force us to have another look at the basics again and again. We may well be just at the beginning of a long and complicated journey.

\section{References}

Asada, K., Nakamura, M., Doi, A., Nagai, H., \& Inoue, M., 2014, ApJ Letters, 781, 2

Begelman, M. C., Blandford, R. D., \& Rees, M. J., 1984, Reviews of Modern Physics, 56, 255

Bicknell, G. V., 1984, ApJ, 286, 68

$\ddagger$ Using the theoretical shape $r_{j} \propto z^{\kappa / 2 \gamma}$ and assuming $\gamma=5 / 3$, we find $\kappa=2.5$ and 1.87 for the near and middle zones respectively. These values seem quite reasonable. 
Cavagnolo, K. W., McNamara, B. R., Nulsen, P. E. J.., Carilli, C. L., Jones, C., \& Brzan, L., 2010, ApJ, 720, 1066

Clausen-Brown, E., Savolainen, T., Pushkarev, A. B., Kovalev, Y. Y., \& Zensus, J. A., 2013, $A \mathscr{E} A, 558,144$

Falle, S. A. E. G., 1991, MNRAS, 250, 581

Fanaroff, B. L. \& Riley, J. M., 1974, MNRAS, 167, 31

Hada, K., Kino, M., Doi, A., Nagai, H., Honma, M., Hagiwara, Y., Giroletti, M., Giovannini, G., \& Kawaguchi, N., 2013, ApJ, 775, 70

Homan, D. C., Lister, M. L., Kovalev, Y. Y., Pushkarev, A. B., Savolainen, T., Kellermann, K. I., Richards, J. L., \& Ros, E., 2015, ApJ, 798, 134

Jorstad, S. G., Marscher, A. P., Lister, M. L., et al., 2005, AJ, 130, 1418

Kaiser, C. R. \& Alexander, P., 1997, MNRAS, 286, 215

Kassim, N., Perley, R. A., Carilli, C. L., Harris, D. E., \& Erickson, W. C., 1996, in Cygnus A Studay of a Radio Galaxy, Proceedings of the Greenbank Workshop, edited by. C.L. Carilli and D.E. Harris, Cambridge University Press, p.182

Kharb, P., Lister, M. L., \& Cooper, N. J., 2010, ApJ, 710, 764

Konigl, A., 1980, PhFl, 23, 1083

Komissarov, S. S., 1990, ApESSS, 171, 105

Komissarov, S. S. \& Falle, S. A. E. G., 1996, ASPC, 100, 173

Komissarov, S. S. \& Falle, S. A. E. G., 1998, MNRAS, 297, 1087

Komissarov, S. S. \& Falle, S. A. E. G., 2003, MNRAS, 343, 1045

Komissarov, S. S., Barkov, M. V., Vlahakis, N., \& Königl, A., 2007, MNRAS, 380, 15

Komissarov S. S., Vlahakis, N., Königl A., \& Barkov, M. V., 2009, MNRAS, 394, 1182

Kovalev, Y. Y., Lister, M. L., Homan, D. C., \& Kellermann, K. I., 2007, ApJ Letters, 668, 27

Laing, R. A. \& Bridle, A. H., 2002, MNRAS, 336, 1161

Lister, M. L., Aller, M. F., Aller, H. D., et al., 2013, AJ, 146, 120

Massaglia, S., Bodo, G., Rossi, P., Capetti, S., \& Mignone, A., 2016, arXiv:1609.02497

Mathews, W. G. \& Brighenti, F., 2003, Ann.Rew.A\&A, 41, 191

Meyer, Eileen T., Sparks, W. B., Biretta, J. A., et al., 2013, ApJ Letters, 774, 21

Moll, R., Spruit, H. C., Obergaulinger, M., 2008, A\&A, 492, 621

Nakamura, M. \& Asada, K., 2014, in The Innermost Regions of Relativistic Jets and Their Magnetic Fields, EPJ Web of Conferences, ed. Jos L. Gmez, v61, id.01004

Nulsen, P. E. J.., Young, Andrew, J. K., Ralph, P., McNamara, B. R., \& Wise, M. W., 2015, IAU Symposium Proceedings, 313, 236

Owen, F. N. \& Ledlow, M. J., 1994, ASP Conference Series, 54, 319

Phinney, E. S., 1983, PhD Thesis, University of Cambridge

Porth, O. \& Komissarov, S. S., 2015, MNRAS, 452, 1089

Pushkarev, A. B., Kovalev, Y. Y., Lister, M. L., \& Savolainen, T., 2009, A\&\&A, 507, L33

de Ruiter, H. R., Parma, P., Fanti, C., \& Fanti, R., 1990, A\&JA, 227, 351

Schreier, E. J., Gorenstein, P., \& Feigelson, E. D., 1982, ApJ, 261, 42

Tchekhovskoy, A. \& Bromberg, O., 2016, MNRAS Letters, 461, 46

Walker, R. C., Ly, C., Junor, W., \& Hardee, P. J., 2008 , JPhCS, 131, 012053 